\title{
Comprehensive investigation of coherent optical OFDM-RoF employing 16QAM external modulation for long-haul optical communication system
}

\author{
Anas Ali Hussien ${ }^{1}$, Adnan Hussein Ali ${ }^{2}$ \\ ${ }^{1}$ Al-Nahrain University, College of Engineering, Computer Engineering Department, Iraq \\ ${ }^{2}$ Middle Technical University, Institute of Technology, Iraq
}

\begin{tabular}{l} 
Article Info \\
\hline Article history: \\
Received Apr 10, 2019 \\
Revised Nov 24, 2019 \\
Accepted Dec 08, 2019 \\
\hline
\end{tabular}

\section{Keywords:}

16QAM

CO-OFDM-RoF

OFDM

RoF

\begin{abstract}
Given the growing need for long haul transmission that requires a high rate of data, the orthogonal frequency division multiplexing scheme (OFDM), is regarded as a technique with high potentials for high-capacity optical networks. OFDM transmits over both optical and wireless channels, with the data distributed over a huge amount of the subcarrier, and the data is distributed over a huge number of subcarriers. OFDM achieves RF signal for a long-haul transmitting by utilizing Radio over Fiber (RoF) system, which is known to produce higher orthogonality of the OFDM modulated signal designed for the wireless network. RoF systems comprise of heterogeneous networks designed through the use of wireless and optical links. The aim of this paper is to carry out an investigation of the performance of the external modulation in RoF links, while analyzing the shortcomings caused by the various elements of the optical system. The Mach-Zehnder modulator (MZM) can be applied in external modulation, and exhibits a more robust performance when implemented with the OFDM modulation technique.
\end{abstract}

Copyright $(2020$ Institute of Advanced Engineering and Science. All rights reserved.

\section{Corresponding Author:}

Adnan Hussein Ali,

Middle Technical University,

Technical Instructors Training Institute,

Baghdad, Iraq.

Email: aaddnnaann63@gmail.com

\section{INTRODUCTION}

Nowadays, Wireless communication system needs growing capacities and comprehensive advantages. Wireless networks, which have become vital in day-to-day activities, are being extensively used in military and civilian applications. Our way of life has been modified by the rapid evolution of communication systems and the rapid advancement of internet; these have led to an increase in the demand for bandwidth [1]. Wireless networks have become so important, such that, without it, it will be impossible for people to transmit vital private information such as control messages, credit card information, e-health data and energy pricing [2].

In other words, reliable mobility and high-transmission bandwidth are required by modern day telecommunication systems. Due to the rapidly growing number of next generation smart mobile devices, and novel bandwidth-intensive user applications like Netflix, Facebook, YouTube, etc., there is an increasing demand for wireless data capacity [3]. With the introduction and evolution of such applications, the way mobile communication systems are used has revolutionized. Due to the advancement in cloud computing/network storage, there is an increasing need for bandwidth of high capacity. The role of wireless communications is crucial in mobile phones and other consumer electronic devices such as tablets, TVs, etc., that need wireless connections with high speed for applications like instant over-the-air back-up, HD-video streaming as well as other multi-media rich applications [4]. 
The main focus of current research in this area is to improve the opto-electric links' efficiency, while providing solutions that are capable of addressing the extant shortcomings present in the entire connection. It is important to find such solutions as the efficiency of the key processes is reduced by such shortcomings. These limitations include the adverse effects of the electron-optical conversions within the transmitter, also the optics-electronic conversions within the receiver side [5]. In addition to these shortcomings, the effect of the fiber optics on the transmitting light wave representing by chromatic dispersion besides the non-linearity, is another shortcoming that the current study seeks to address. Some of the problems that have been associated with the wireless system are transmission losses and atmospheric reduction, and that is why the use of Radio over Fiber system is employed to solve the problem [6].

In this paper, the attributes and performance of Orthogonal Frequency Division Multiplexing (OFDM) as a technique of modulation for a RoF are investigated. The study basically aims at studying the efficiency of the external and direct intensity in RoF link, while analyzing the setbacks caused by the various elements of the optical system. This paper investigates the backbone transmission technique of an OFDM-RoF system as for the Long-Haul Optical Communication System, the proposed model employs RoF system with OFDM-16 QAM technique and coherent optical orthogonal frequency division multiplexing (CO-OFDM) transmission technology.

\section{EASE METHODS AND MATERIALS}

\subsection{Rof}

For the first time in 1990 the use of RoF was employed in cordless and mobile telephone services. With RoF, the use of highly linear optic fiber links is employed in establishing a connection between Control Stations (CS) and Base Station (BS). The aim of this is to enhance the distribution of RF signal between the Base Stations and Control Stations [7]. The processes of coding, modulation, routing and demodulation are implemented at the Control Station, while the signal conversion of optical to an electrical, or conversely is implemented in the Base Station.

Basically, RoF is made up of every kind of hardware that supports the foisting of RF signal on the optical carriers concerned a transmitter side. More so, to enable the distribution of signal from Control Station to Base Stations, a fiber optic link is required. In order to enable the recovery of signal from the carrier side, all the hardware are needed at the receiver side. The RoF system is a good mixture of radio waves and fiber optic link. This wireless network technology can be regarded as the next generation of broadband wireless communication that is capable of transmitting data with high speed, while increasing the capacity channel of RF frequency modulation [8].

With the new telecommunication systems, mobility that is reliable alongside high-transmission of bandwidths are required. In order to achieve these, the use RoF can be employed because it's capable of fulfilling these requirements through the use of the large amount of bandwidth provided by optical communications systems and the flexibility offered by the wireless systems. Due to the ability of the system to use optical fiber for the transmission of millimeter and microwave for short and long distance, it is regarded as a suitable wireless access network technique [9]. In addition to this, WLAN, fourth and fifth generation mobile networks can be supported by this technology.

RoF consists of two sides that connect to a fiber optic link, and the two sides are referred to as remote site (RS) and central site (CS). They are responsible for the transmission of the radio frequency signal uplink and downlink. The transmission of optical signal between the RS and CS is executed by the RoF network within the optical band passes $[10,11]$. Figure 1 shows the RoF model with the base station as the point for conversion from optical to electrical and vice versa.

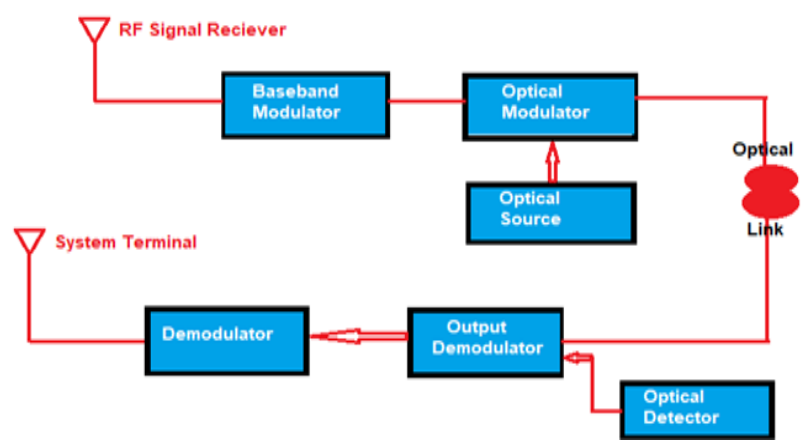

Figure 1. Block diagram of RoF system 


\subsection{Optical transmission link 2.2.1. Optical fiber}

The Optical fiber can be considered as a platform or a medium of conveying light information form from one point to the other. This implies that the optical fibre serves as a wavelength that enables the distribution of light from one point to the other. The fiber is a thin glass filament that is capable of providing a route for the transmission of light. However, it incurs some losses as a result of complete reflection from within.

Optical fiber provides two low attenuation regions, whereby one of them is at approximately $1300 \mathrm{~nm}$ with an attenuation that is below $1 \mathrm{~dB} / \mathrm{km}$ with a bandwidth $25 \mathrm{THz}$. On the other hand, the other region starts with $1550 \mathrm{~nm}$ approximately, which has less than $0.2 \mathrm{~dB} / \mathrm{km}$ attenuation, and a $25 \mathrm{THz}$ bandwidth [12]. This two regions combination can be made a total bandwidth of $50 \mathrm{THz}$. As a result of the low attenuation regions, there will be minimal loss of signal when data is being transmitted. The implication of this is that, so many repeaters and amplifiers will be unnecessary and not required.

There are basically three kinds of optical fibre in a communication system, and they include stepindex single mode, step-index Multimode and graded index [13]. The measurement of the Step-index Multimode fiber, can take from cladding to core because it acts as a refraction profile index which steps from high to low and vice versa. For a single mode with step index, just one path allows the travelling of light within the fibre. While Graded index single-mode fiber type offers a large core diameter with high bandwidth.

\subsubsection{Optical transmitters}

The highest widespread light sources in fiber optical communications are LED and laser diode. These devices offer the advantage of output power for a wide range of applications. The power can also be directly modulated where the input current varies across the devices. More so, they are compatible with optical fiber and are highly efficient [14]. The difference between them is concerning a coherent output light from the laser diodes where the production of optical energy can be produced from an optical resonant cavity [15].

\subsection{OFDM}

The OFDM possess very high spectrum data efficiency. It is designed for improving the capability of the system and the transmitted distance over optical fiber and RF. It can be regarded that OFDM is a modulation method that was great potential benefits for optical wireless system in the future [16]. OFDM is an effective method of solving the problem of inter-symbol interference (ISI) caused by the spread of wireless channels in wideband signaling over multipath radio channels [17]. With this method, data is transferred in a parallel manner over an amount of narrowband flat subchannels.

OFDM, which is perceived as a technology with great potential benefits for high-speed wireless and optical transmission. OFDM is a technology that supports multicarrier transmission, in which high-speed data stream is transmitted by dividing it into several parallel channels of low-speed data. The OFDM in wireless systems is the first technology at physical layer that emerged, and is presently being adopted in many recent wireless systems such as WiMAX, LTE, Wi-Fi [18].

The formation of OFDM signal involves using inverse fast Fourier transform (IFFT), with the addition of a cyclic extension for getting spectral modulator [19]. Current signal modulation is sent in the formats of QAM and QPSK from the OFDM transmitter to IFFT transformation. Therefore, the next level requirement in the OFDM system is sent to the D/A converter. In the side of OFDM receiver, the data are sent in the demodulator format, which is reverse of IFFT (FFT transformation). A final RF signal at the receiver is demodulation form and then transmitted to the users as shown in Figure 2.

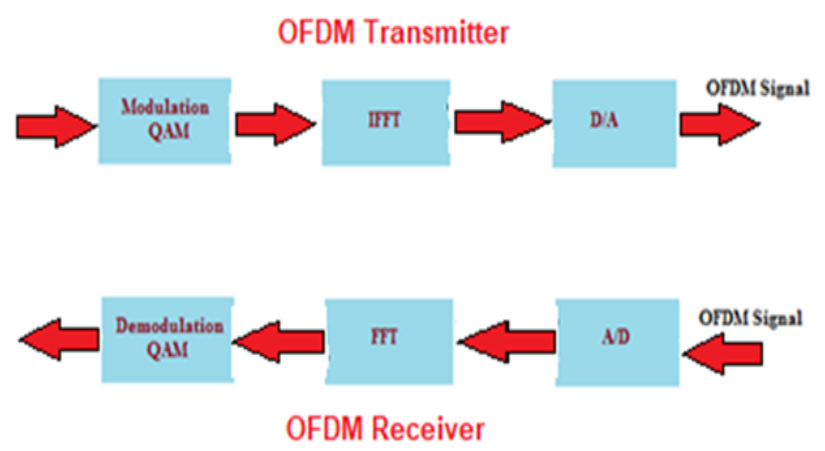

Figure 2. OFDM transmitter and receiver 
Due to the ability of OFDM to provide large data rates with adequate capabilities of handling impairments of radio channel, it is rapidly emerging as a preferred technique for wireless communication modulation. In the OFDM transmission scheme, the use of many sub-carriers are employed in converting high rate serial data to low rate multiple parallel data streams. This process leads to the prolongation of the duration of the symbol, thereby facilitating the elimination of Inter Symbol Interference (ISI) [20]. A Multimode or single mode optical fiber serves as an approach of transportation between the base stations and the antenna, in which a frame of electronics may be situated.

The provision of multi-path spread spectrum by OFDM modulation technique for the enhancement of mobility makes it a technology that is capable of supporting future broadband wireless communication. More so, the OFDM is considered as an entirely different innovation in terms of the approach used for system designing because it combines multiple-access and modulation for channels of communication. In addition, with this technology, the spectrum is split at one user's end. This is referred to as single frequency for a unique operator. OFDM can be considered as a kind of frequency division multiplexing (FDM) which is orthogonal, thereby being able to share all channels using a single frequency. With OFDM, the spread range is allowed to overlap OFDM allows the overlapping of spread spectrum, for the reason that they are orthogonal besides they cannot interfacing with each other's as shown in Figure 3.

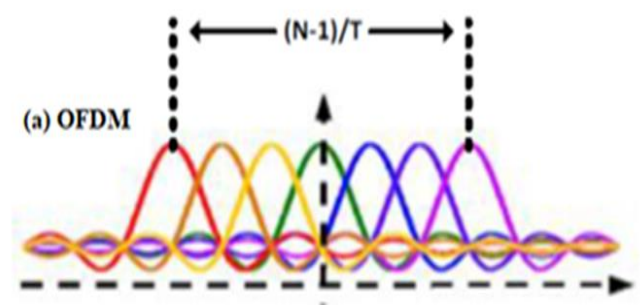

Figure 3. OFDM frequency spectrum

As mentioned previously, OFDM allows the use of multiple carriers that have equal frequency spacing for the formation of parallel transmission of data [21]. The efficiency of bandwidth can be enhanced when the spacing between the subcarriers within the OFDM system is reduced [22]. It is based on the principle of OFDM that the Fast-OFDM operates, and offers two times the efficiency of bandwidth which OFDM offers, where the separation in frequencies of the sub-carriers can be $(1 / 2 \mathrm{~T}) \mathrm{Hz}$, where, $\mathrm{T}$ is the time interval of the subcarriers. This meaning that the same rate of data as that of an OFDM is produced by the Fast-OFDM system [23]. The Fast-OFDM is able to do so using just half of the bandwidth used by the traditional OFDM as presented in Figure 3.

Many advantages of OFDM like the achieving of higher data rate in wireless channels with conveniently implementation by applying IFFT and FFT procedures, also OFDM have well tolerances for inter-symbol interference (ISI) [24]. Other advantages of OFDM include easy adaptability to unfavorable channel conditions without sophisticated equalization, robustness against the interferences of narrow-band co-channel, strong against inter-symbol interference besides fading which resulting from multipath propagation, well-organized implementation using FFT, high spectral efficiency, low sensitivity for errors at time synchronization [25]. Furthermore, with OFDM, there is no need for Tuned sub-channel receiver filters (as in conventional FDM). Lastly, through the use of OFDM, Single Frequency Networks are facilitated, i.e. transmitter macro diversity.

\section{OFDM SYSTEM DESCRIPTION}

In Figure 4, the block diagram of transmission system using OFDM is illustrated. As illustrated in Figure 4, the high rate stream of digital data at the transmitter is divided into $\mathrm{N}$ parallel streams with some schemes of modulation (QAM, PSK etc) used to map each of the streams to a symbol stream. The use of inverse discrete Fourier transforms (IDFT) is employed in modulating the symbols onto the subcarriers. The main function of the IDFT is to transform the OFDM symbol from frequency to time domain. The functions of the inverse fast Fourier transform (IFFT) and an IDFT are similar, just that the IFFT is much more efficient in terms of computation. After the IDFT operation, an addition of a cyclic prefix is made to the OFDM symbol before digital-to-analogue converter (DAC) output is obtained. The DAC output is a baseband analog signal which is then up-converted in frequency and transmitted. 


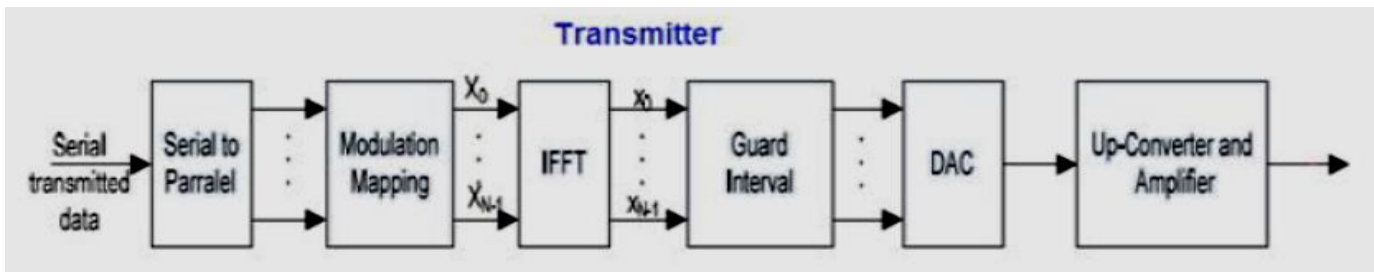

Figure 4. Block diagram showing a basic OFDM transmitter system

\subsection{OFDM-RoF system}

The combination of OFDM and RoF system (OFDM-RoF) is considered for future broadband wireless communication. With the RoF system, wider coverage and higher speed more than that provided by other systems are offered. There are two main parts of the OFDM-RoF system model, and they are RF-OFDM receiver and RF-OFDM transmitter as shown in Figure 5.

By means of the RoF system, the OFDM the modulation technique can be increased, and it is also able to deal with the different shortcomings of the wireless transmission, like dispersion of chrome, attenuation of electrical power and phase modulated through the optical link. Many advantages can be achieved from the system combinations here for the data transmission system with future high-speed. As observed in Figure 5, LiNb (Lithium Niobate) Mach-Zehnder modulator is used for both Electrical to Optical (E-O) and Optical to Electrical (O-E) conversions.

Figure 5 describes an OFDM-RoF system's block diagram. From the illustration, the input of the digital data is initially split into parallel streaming data from OFDM transmitter, after that these data is passed on the link of optical fiber. Within this link, a continuous wave (CW) laser (transmitter) will emit a continuously beam like a train of short laser pulses. At the MZM (external modulator), the signals of electrical waves acquired by OFDM transmitter combine with a continuously wave light from laser, then they have been modulated by MZM for creating an optical signal that may be sent through the optical fiber.

When the signal is optically produced in the fibre, the receiving data is detected with a photodetector (receiver). A photodetector is stream converting the incoming photonics to the original stream of electrons, which means a back conversion of electrical signals from the optical signals. In order to get back the initial data, a recombination of signal occurs within the OFDM receiver. The system can be enabled for use in both long-haul and short distance transmission at extremely high rates of data by combining OFDM with RoF. With this, ultra-wide band access and RF signals of large capacity can be obtained.

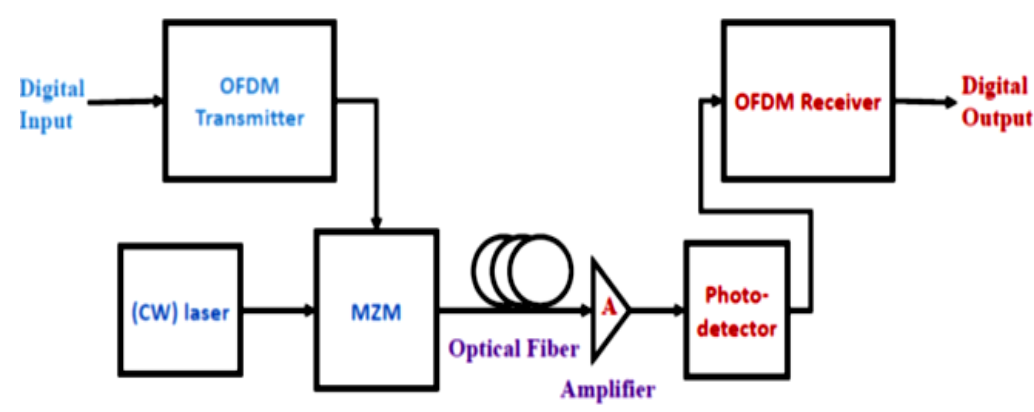

Figure 5. Block diagram of OFDM - RoF system

\subsection{OFDM- RoF methodology}

The procedure used in the design of the OFDM-RoF system involves of adaptation with Optisystem software, component generation for the designing system, simulated of objects, then a simulation running and data analysis. The use of Optisystem is employed in designing, constructing and simulating the RoF topology. A comprehensive software is an Optisystem software that offers a platform for planning, testing and simulating the optically links within the modern networks of optical layers. More so, Optisystem is a simulation package used in optically communication systems for designing, testing and optimizing a wide range of optical links within a physical layer of varieties optical networks, ranging from an analog video broadcast up to intercontinental backbones. 
The simulation environment of the Optisystem, being a system level simulator based on real-time fiber optic communication systems modelling, is powerful and totally hierarchical in terms of system sound components. The expansion of its capabilities can be carried out with ease, allowing the adding of other components and less sophisticated interfaces to a variety of using tools. The main aim of this study is to model and simulate an OFDM-RoF system with a length of fiber up to $100 \mathrm{~km}$ utilizing 16-Quadrature Amplitude Modulation (16-QAM).

In comparison to binary shift keying (BPSK), 8-QAM and 4-QAM, High-level modulation like 16-QAM is more efficient in terms of spectral signal, although the robustness of the system decreases as the increasing of symbol data modulation. Furthermore, when Mach-Zehnder modulator is used like an external modulator, it exhibits more robust performances in comparison to the directly modulated if an implementation of the other OFDM modulation techniques. In the simulation of the Optisystem software, an RF signal can be passed into the RoF system, therefore it can convert optical signals so as to increase the channel capacity.

\section{PROPOSED OFDM ROF SYSTEM MODEL}

The Proposed model dealing with RoF system integrating with OFDM-16 QAM technique comprises of five parts namely RF-OFDM transmitter, RF to optical up-converter (RTO), optical link, optical to RF down-converter (OTR), and RF OFDM receiver. Figure 6 presents the model. The results of simulation are visualized using spectrum analyzer for both RF and optical signals, while meters for electrical and optical power, as well as constellation visualizer can be applied for displaying the output spectrum of the components.

\subsection{RF-OFDM transmitter}

The transmitter part contains a QAM sequence for generating an OFDM signal bit sequences that have four bits/symbol and followed from different frequencies coming with each subcarrier and considered part of all the 16 subcarriers. When the OFDM modulation is at 16-QAM position, which is also known as QAM M-array position, it produces 512 subcarriers and 1024 FFT points. In this system, the use of OS12 is employed. OS12 is one of the components of OFDM in which the outputs I and Q are directed towards an LP-CROF. In the LP-CROF, it is possible to arrange the roll off factor value from 0 to 1 . The performance of the system can be significantly influenced by the Roll Off Factor and it is capable of determining the complexity of the receiver. When an electric gain is utilized prior to its arrival to the optical modulator, it causes power loss. The structure of RF-OFDM transmitter's simulation can be shown in Figure 6.

\subsection{RF to optical upconverter (RTO)}

This is an E/O step conversion, which involves the use of optical LiNb MZM as a modulator for the system model. One of the most essential parts of Lightwave systems with high bit rate is LiNb MZM, which is an external modulator that is fixed between laser and RF. Here, laser with continuous wave can be used for delivering signals of continuous optic waves.

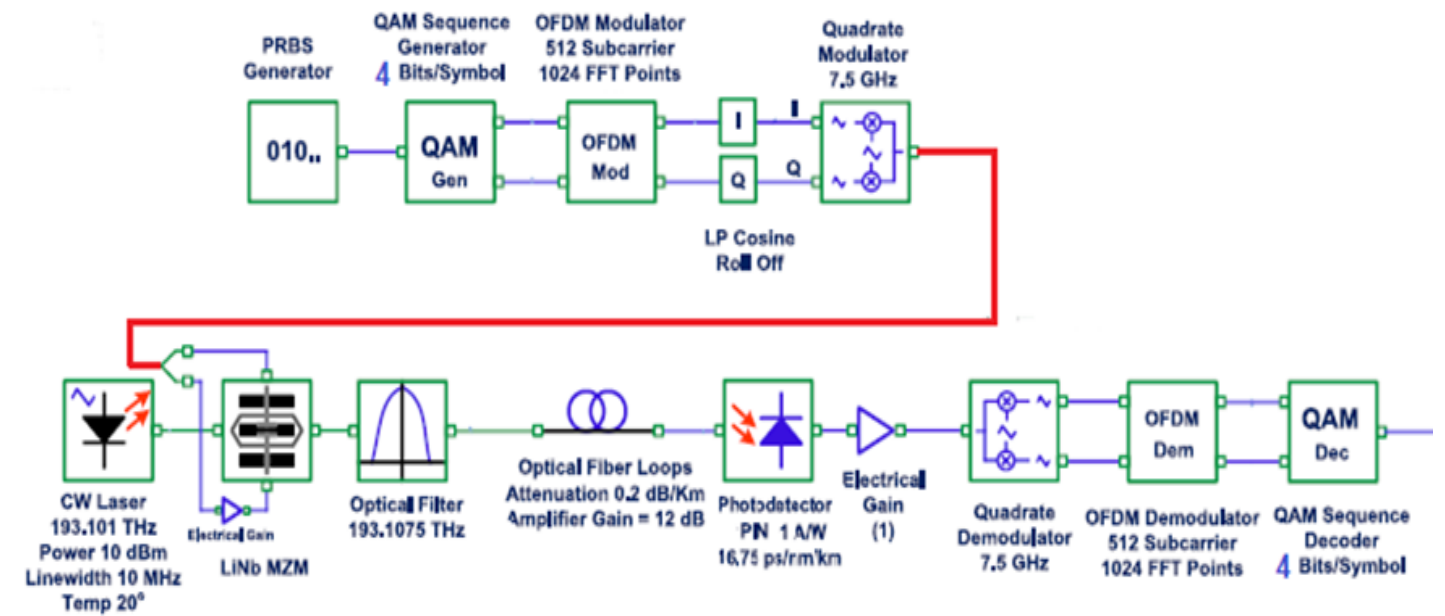

Figure 6. External modulation of 16-QAM OFDM 


\subsection{Optical link}

When this system is designed, the use of loop control is employed for a long fiber optic variation having 200 wavelength/time. The losses which the scattering of noise fiber as well as dispersion of signal cause are anticipated with the aid of an Optical Filter Frequency. The optical fiber length is simulated is 10 to $100 \mathrm{~km}$.

\section{OFDM ROF OPTISYSTEM SIMULATION MODEL}

The Optisystem software is utilized for simulating the optical communication experiments. The use of visualizing tools like an optical spectrum analyzer, a constellation visualizer and an RF spectrum analyzer are employed in visualizing the performances of the OFDM-ROF system. They are mainly used for displaying the output circuit components spectrum. Figure 7 shows a circuit of 16-QAM OFDM external modulation with the recovering and modulating signals. In QAM-OFDM, M-Array sequences with I and Q outputs are produced by feeding the sequences of random bits into the M-QAM generator. Afterwards, the QAM symbols are transmitted over parallel overlapped orthogonal subcarriers by feed the OFDM modulator with I and $\mathrm{Q}$ outputs. Then the I and Q electrical inputs are modulated by $7.5 \mathrm{GHz}$ RF carriers through the use of a quadrature modulator. The main structural parameters that were used in this simulation are specified as follows: A Bit rate of $10 \mathrm{Gbps}$, the time window was $1.6 \mu \mathrm{s}$, the Sampling rate of $40 \mathrm{GHz}$, the sequence length was 16384 bits, while the sample per bit is 4 and finally, the number of samples 65536 .

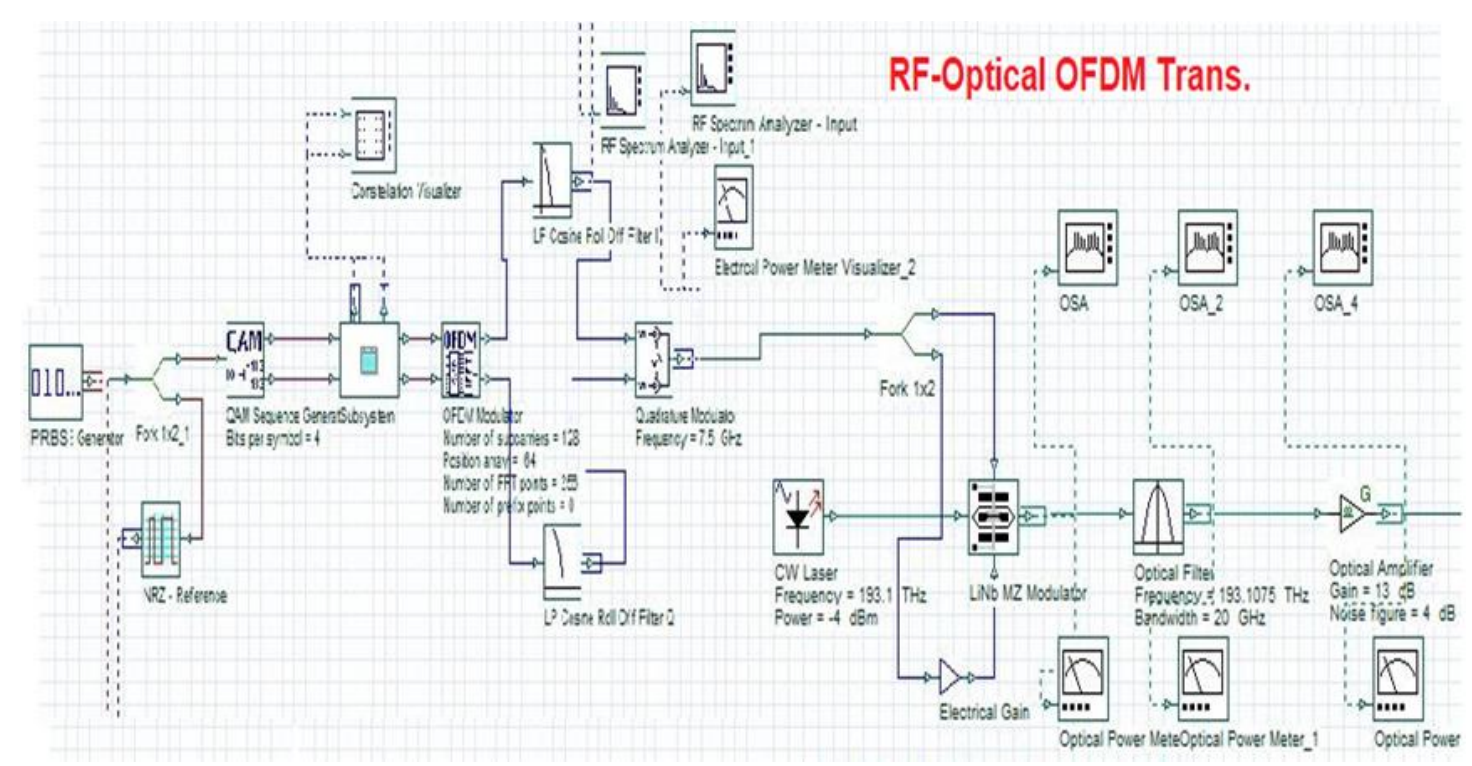

Figure 7. Optisystem simulation of Transmitter

\section{RESULTS \& DISCUSSION OF OFDM RoF SYSTEM}

The Optisystem software was utilized for simulating an experiment of the optical communication. In 16QAM-OFDM-RoF transmitter system, the bit generator can be used in generating the NRZ form signal sequences of 0 and 1 with 16384 bits, and such bits are encoded with 16QAM decoder as shown in Figure 8(a), which uses 4 bits per symbol. The result of the RF spectrum and the optical spectrum modulation of the transmitting intensity modulation signals can be considered double-sideband (DSB) optical carrier as shown in Figure 8(b). This RF OFDM signal at that point as shown in Figure 6 are utilized to obtain an LiNb MZ Modulator. The output of the MZM is a symmetric optical spectrum that is centered around $193.1 \mathrm{THz}$ due to the CW laser signal shown in Figure 8(c). This spreading optical spectrum over a wide frequency ranges, thereby affecting the signal quality when transmitting over the optical fiber link. The modulated signal at both time and frequency domain is shown in Figure 8(d). The power of the modulated OFDM signal is equal -6 $\mathrm{dBm}$ and bandwidth of $15 \mathrm{GHz}$, while the main OFDM part of the signal is $3 \mathrm{GHz}$ from two sides of the frequency centre. The final operation of the transmitter is the upconversion of RF OFDM signal to optical signal at $1550 \mathrm{~nm}$ window (193.1 THz) by using an external modulator of MZM with a small power value of $-8 \mathrm{dBm}$. The resulting time and frequency domain are shown in Figure 8(e). 

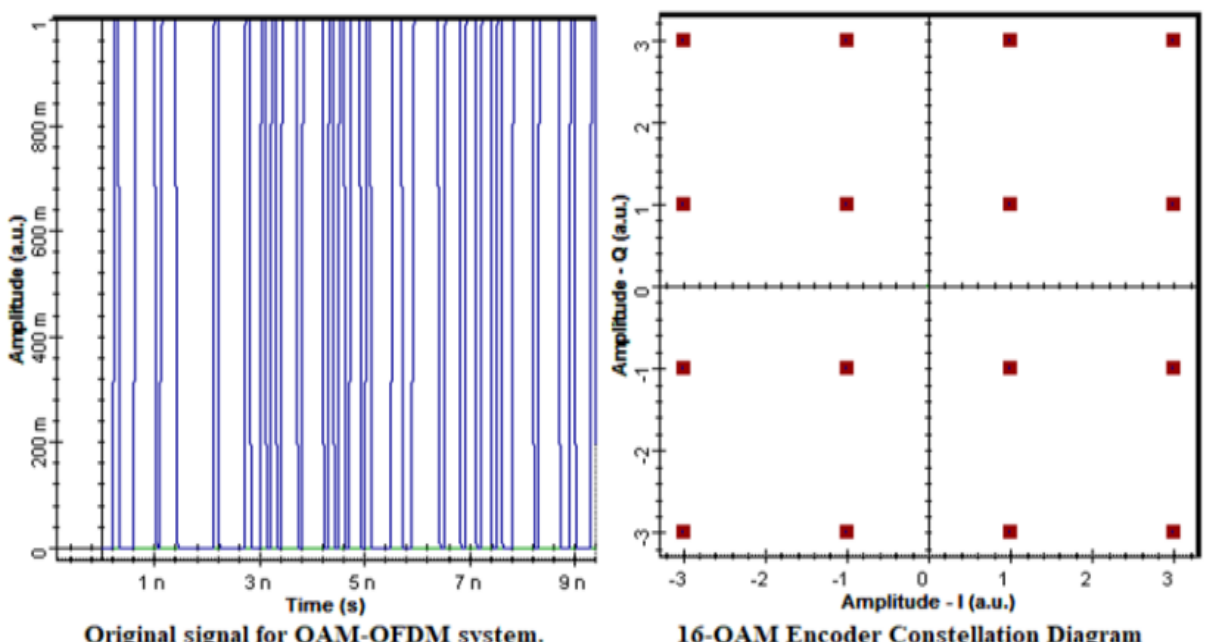

(a)
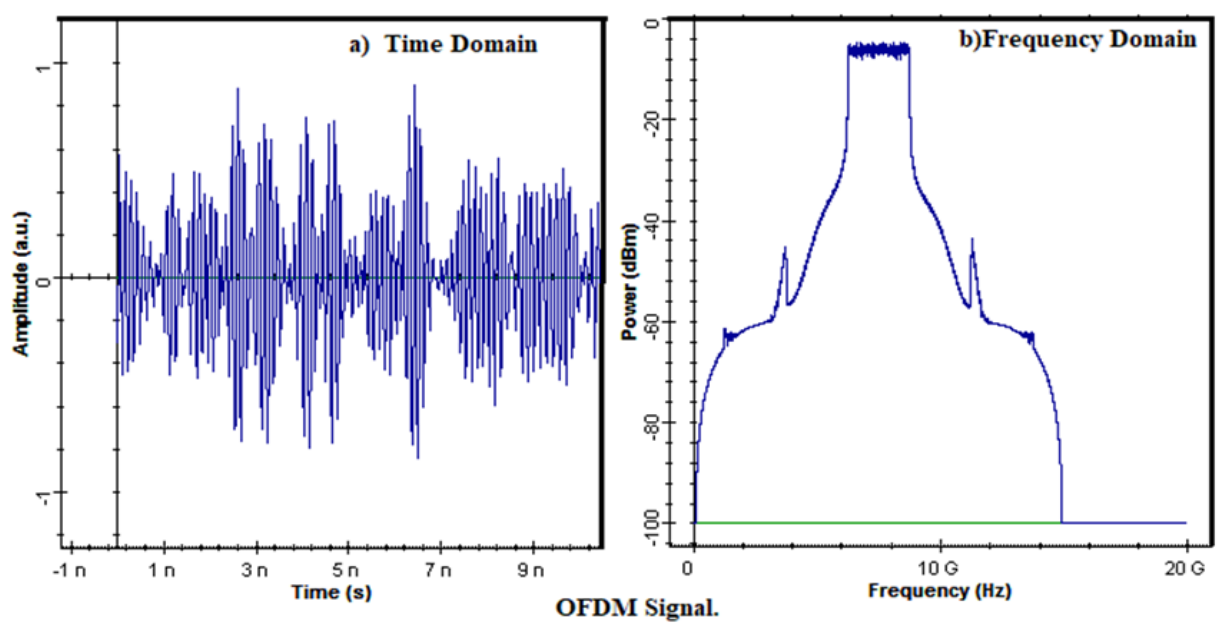

(b)

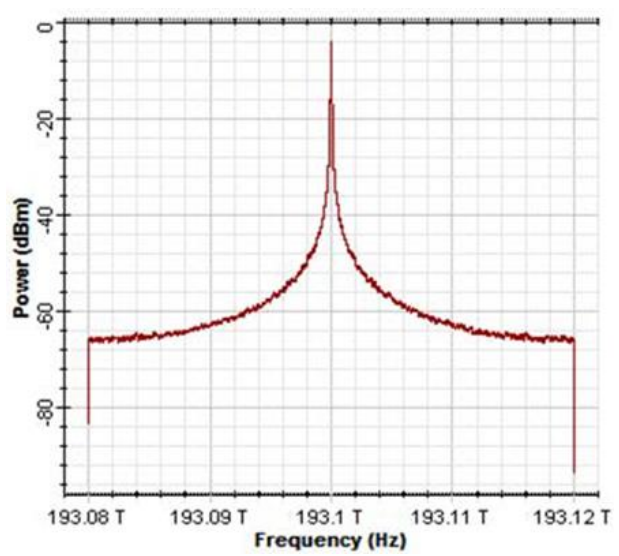

(c)

Figure 8. (a) 16QAM-OFDM-RoF system, (b) RF OFDM signal,

(c) CW laser signal centered around $193.1 \mathrm{THz}$ 

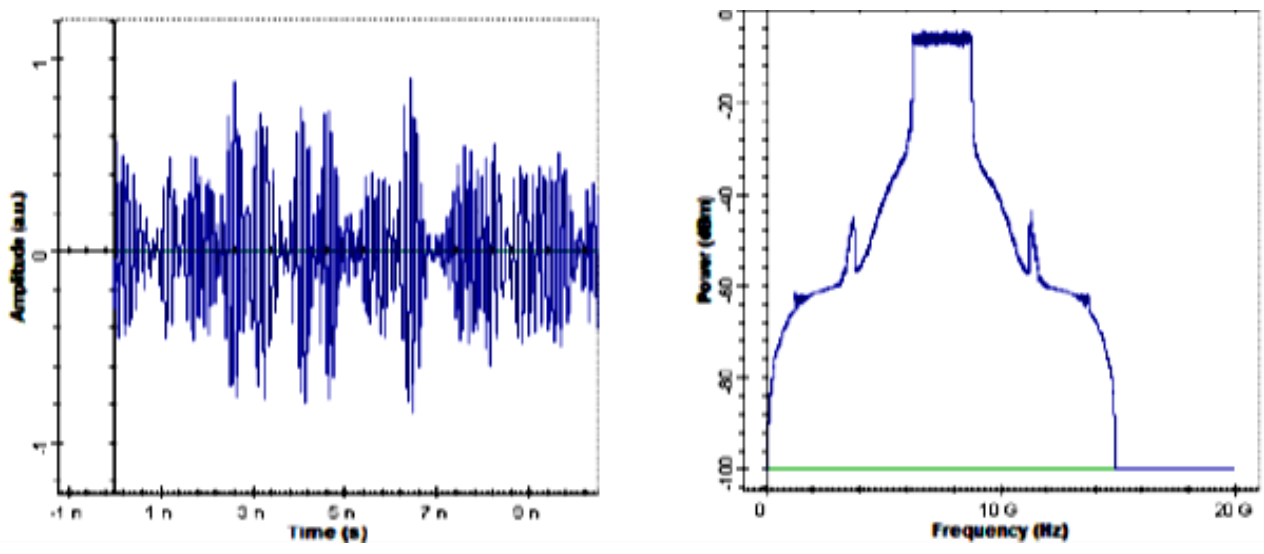

(d)
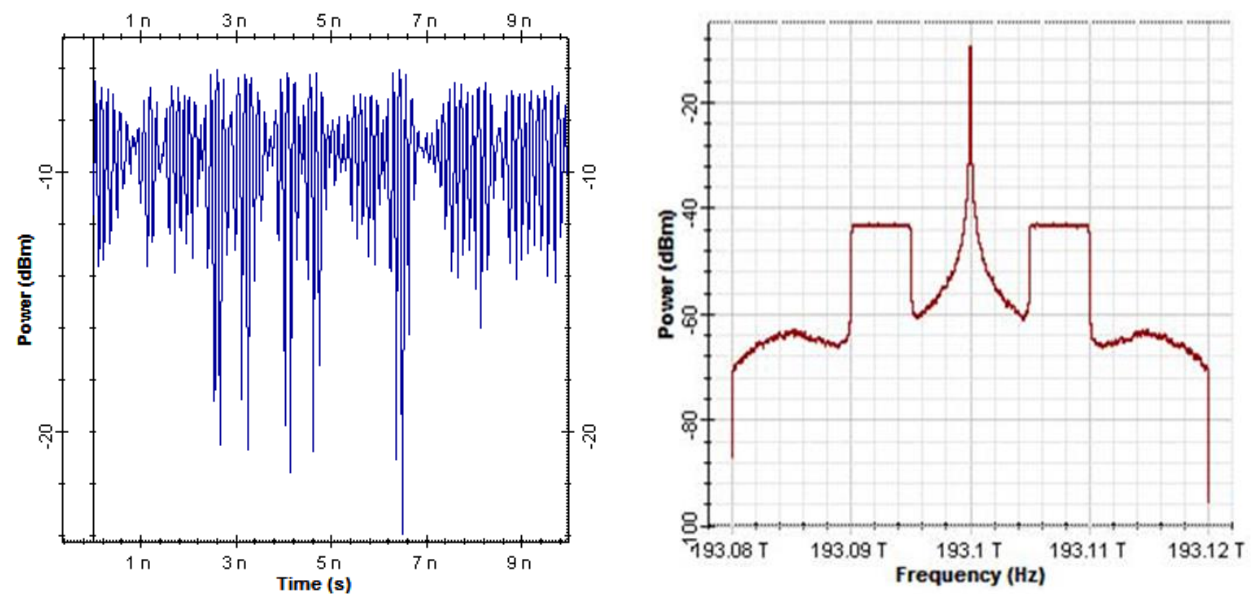

(e)

Figure 8. (d) The modulated OFDM signal, (e) Optical signal from external MZM modulator

So, the DSB composite signal is modulated onto an optical carrier signal of frequency 193.1 THz. Thus, the option of a spectrum enhancement is needed by an optical amplification due to the poor quality OFDM spectrum resulting from the baseband and the transmission path. Then, when an optical DSB signal is propagated over the fiber optics, an establishment of relative phase difference between the double-sides band with optical carrier due to chromatic dispersion which occurs in the fiber.

\section{CONCLUSION}

This study briefly described and explained the central idea of using QAM technique to generate the OFDM symbols, which were also highlighted and discussed. More so, a discussion on the OFDM-RoF system model alongside all its components which include transmitter, receiver and optical link was presented in this work. The OFDM-RoF systems with external intensity modulation had been accessible and then compared. It was observed that better performance was exhibited by the external modulation method, which also demonstrated the ability to yield a symmetric spectrum that is stable. The viewing values obtained that most better modulation performances are achieved by the external MZM. With the benefits of the OFDM and RoF combination, both short distances as well as long haul transmission can be applied with maximum high data rates. The optical OFDM RoF system have been modeled for many applications, but in this study, it is used as frequency carrier 7.5 GHz. The system data rate is 10 Gbps with 16QAM modulation type and different number of subcarriers. Based on the result of this study, it is easy for orthogonality maintaining of OFDM signal in 16QAM formatting at data bit rate of 10Gbps. The model produced using these techniques van be very useful in increasing the RF signals quality in current and next generation of wireless optical communication system. 


\section{REFERENCES}

[1] R. M. S. Arun and H. Mascreen, "Analysis of OFDM Scheme for RoF System and Characterization with GPON Architecture," Int. Journal of Engineering Research \& Technology (IJERT), vol. 4 no. 01, pp. 441-444, 2015.

[2] R. C. Alferness, "The evolution of configurable wavelength multiplexed optical networksVA historical perspective," Proc. IEEE, vol. 100, no. 5, pp. 1023-1034, May 2012.

[3] Adnan H. Ali, Farhood, A. D., "Design and Performance Analysis of the WDM Schemes for Radio over Fiber System with Different Fiber Propagation Losses," Fibers, vol. 7, no. 19, 2019.

[4] A. E. Willner, S. Khaleghi, M. R. Chitgarha, and O. F. Yilmaz, "All-optical signal processing," Journal of Lightwave Technology, vol. 32, no. 4, pp. 660-680, Feb. 15, 2014.

[5] Y.-K. Wong, S. M. Idrus, and I. A. Ghani, "Performance Analysis of the OFDM Scheme for Wireless over Fiber Communication Link," International Journal of Computer Theory and Engineering, vol. 4, no. 5, Oct. 2012.

[6] J. Ma, "Dual-tone QPSK optical millimeter wave signal generation by frequency-nonupling the RF signal without phase precoding," in IEEE Photonics Journal, vol. 8, no. 4, pp. 1-7, Aug. 2016.

[7] Faris M. Ali, Eman A. Abd Ali, and Mayada G. Tarbul, "Performance Analysis of Radio over Optical Fiber System with OFDM Using Multiplexing Techniques," International Journal of Applied Engineering Research, vol. 13, no. 12, pp. 10831-10844, 2018

[8] X. Li, J. Yu, J. Zhang, J. Xiao, Z. Zhang, Y. Xu, and L. Chen, "QAM vector signal generation by optical carrier suppression and precoding techniques," IEEE Photonics Technol. Lett., vol. 27, no. 18, pp. 1977-1980, 2015.

[9] A. J. Lowery, "Fiber nonlinearity mitigation in optical links that use OFDM for dispersion compensation," IEEE Photonics Technology Letters, vol. 19, no. 19, pp. 1556-1558, Oct. 2007.

[10] Al-Raweshidy, H., and Komaki, S., "Radio over fiber technologies for mobile communications networks," Artech House Publishers, Jan. 2002.

[11] Adnan H. Ali, "Simultaneous measurements for tunable laser source linewidth with homodyne detection," Computer and Information Science, vol. 4, no. 4, Jul. 2011.

[12] M. M. Kareem, M. Ismail, M. A. Altahrawi, N. Arsad, M. F. Mansor, and A. H. Ali, "Grid Based Clustering Technique in Wireless Sensor Network using Hierarchical Routing Protocol," in 2018 IEEE 4th International Symposium on Telecommunication Technologies (ISTT), pp. 1-5, 2018.

[13] Sinan A. Khwandah, J. P. Cosmas, Ian A. Glover, P. I. Lazaridis, Neeli R. Prasad, and Zaharias D. Zaharis, "Direct and External Intensity Modulation in OFDM RoF Links," IEEE Photonics Journal, vol. 7, no. 4, Aug. 2015.

[14] R. Karthikeyan and S. Prakasam, "A Survey on Radio over Fiber (RoF) for Wireless Broadband Access Technologies," International Journal of Computer Applications (0975-8887), vol. 64, no. 12, Feb. 2013.

[15] F. Almasoudi, K. Alatawi and M. Matin., "Study of OFDM Technique on RoF Passive Optical Network," Optics and Photonics Journal, vol. 3, no. 2, pp. 217-224, 2013.

[16] W. Xu, M. Wu, H. Zhang, X. You, and C. Zhao, "ACO-OFDM-specified recoverable upper clipping with efficient detection for optical wireless communications," IEEE Photonics Journal, vol. 6, no. 5, pp. 1-17, Oct. 2014.

[17] N. Veneetha, K. Joseph and R. Asha, "Performance analysis of direct detection and coherent detection system for optical OFDM using QAM, and DPSK," IOSR Journal of Engineering, vol. 7, Jul. 2013.

[18] Yuming Xu, Xinying Li, Jianjun Yu, Gee-Kung Chang, "Simple and reconfigured single-sideband OFDM RoF system," OPTICS EXPRESS, vol. 24, no. 20, Oct. 2016.

[19] Yoon-Khang Wong, S. M. Idrus, and I. A. Ghani, "Performance Analysis of the OFDM Scheme for Wireless over Fiber Communication Link," Int. Journal of Computer Theory and Engineering, vol. 4, no. 5, Oct. 2012.

[20] M. Xue, S. Pan, and Y. Zhao, "Optical Single-Sideband Modulation Based on a Dual-Drive MZM and a $120^{\circ}$ Hybrid Coupler,” Journal of Lightwave Technology, vol. 32, no. 19, pp. 3317-3323, 2014.

[21] Adnan H. Ali, Hayder J. Alhamdane, Begared S. Hassen, "Design analysis and performance evaluation of the WDM integration with CO-OFDM system for radio over fiber system," Indonesian Journal of Electrical Engineering and Computer Science, vol. 15, no. 2, pp. 870-878, Aug. 2019.

[22] F. H. Partiansyah, A. Susanto, I. W. Mustika, S. M. Idrus, and S. H. Purnomo, "Dithering Analysis in an Orthogonal Frequency Division Multiplexing-Radio over Fiber Link," International Journal of Electrical and Computer Engineering (IJECE), vol. 6, no. 3, pp. 1112-1121, 2016.

[23] Ali, A. H., Abdul-Wahid, S.N., "Analysis of self-homodyne and delayed self-heterodyne detections for tunable laser source linewidth measurements," IOSR J. Eng., vol. 2, no. 10, Oct. 2012.

[24] Ahmed J. Abid, Fawzi M. Al-Naima, and Adnan H. Ali, "Comprehensive Modeling of Photovoltaic Array based on Proteus Software," International Journal of Applied Engineering Research, Vol. 13, No. 6, pp. 4440-4447, 2018.

[25] Ali A. Abdulrazzaq, Adnan H. Ali, "Performance Investigation of Grid Connected Photovoltaic System Modelling Based on MATLAB Simulation," International Journal of Electrical and Computer Engineering (IJECE), Vol. 8, No. 6, pp. 4847-4854, 2018. 